\title{
Le sida au Canada - Rapport de surveillance 2016
}

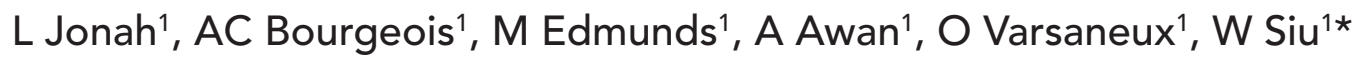

\section{Résumé}

Contexte : Bien qu'il y ait encore une épidémie mondiale de personnes touchées par le virus de l'immunodéficience humaine $(\mathrm{VIH})$, le nombre de personnes qui décèdent du syndrome d'immunodéficience acquise (sida) a diminué, en grande partie grâce au succès du traitement antirétroviral.

Objectif : Fournir un aperçu détaillé des cas de sida signalés au Canada en cernant les tendances selon l'emplacement géographique, le sexe, le groupe d'âge et la mortalité. Alors que l'analyse descriptive se concentre sur l'année 2016, les résultats sont présentés pour les cas signalés depuis le début de la surveillance nationale du sida en 1979.

Méthodologie : L'Agence de la santé publique du Canada (ASPC) surveille le sida au Canada par l'intermédiaire du Système national de surveillance du VIH/sida et de Statistique Canada. Le Système national de surveillance du VIH/sida est un système de surveillance passif, basé sur des cas, qui conserve des données non nominatives sur les cas de VIH et de sida fournis volontairement par les provinces et les territoires du Canada. Il convient de noter que le sida n'est plus une maladie à déclaration obligatoire à Terre-Neuve-et-Labrador (depuis 2009) et à l'île-du-Prince-Édouard (depuis 2012). Des données ont également été récupérées, basées sur les décès annuels attribués au VIH/sida par Statistique Canada. Les données ont été rassemblées, les tableaux et les figures préparés, puis les statistiques descriptives appliquées par I'ASPC et validées par chaque province et territoire.

Résultats : Un total de 114 cas de sida ont été déclarés en 2016, avec un total cumulé de 24179 cas depuis 1979. Ces nombres représentent une baisse constante du nombre de cas de sida déclaré par année de diagnostic au Canada depuis 1993. Parmi les provinces qui ont déclaré des résultats, les plus grands nombres de cas en 2016 ont été déclarés par l'Ontario, la Saskatchewan et l'Alberta. Les hommes représentaient $72,8 \%$ des cas de sida déclarés, et les adultes de 50 ans et plus représentaient la plus grande proportion selon le groupe d'âge $(36,0 \%)$. Pour toutes les années de l'article combinées, la répartition par âge des cas de sida est similaire selon le sexe, bien qu'une plus grande proportion de femmes aient moins de 30 ans. Des données limitées ont été rapportées sur l'ethnicité et les facteurs de risque. Le nombre de décès annuels attribués au sida a diminué depuis 1995. Il y a eu un faible nombre record de 241 décès liés au sida en 2013, I'année la plus récente pour laquelle des données étaient disponibles. Le nombre de décès liés au sida au Canada a diminué de $86,2 \%$ depuis 1995.

Conclusion : Le nombre de cas de sida déclarés par les provinces et les territoires participants et le nombre de décès liés au sida déclarés par Statistique Canada ont diminué. Bien que cela représente une évolution encourageante, les données doivent être interprétées avec prudence compte tenu des limites de l'ensemble de données, ce qui pourrait entraîner une sous-estimation de l'ampleur de la maladie.

\begin{abstract}
Affiliation
${ }^{1}$ Centre de la lutte contre les maladies transmissibles et les infections, Agence de la santé publique du Canada, Ottawa (Ontario)
\end{abstract}

*Correspondance : HASS@ phac-aspc.gc.ca

Citation proposée : Jonah L, Bourgeois AC, Edmunds M, Awan A, Varsaneux O, Siu W. Le sida au Canada Rapport de surveillance 2016. Relevé des maladies transmissibles au Canada. 2017;43(12):292-7.

https://doi.org/10.14745/ccdr.v43i12a02f

\section{Introduction}

Le virus de l'immunodéficience humaine $(\mathrm{VIH})$ et le syndrome d'immunodéficience acquise (sida) constituent une épidémie mondiale continue. Il y a eu d'énormes progrès causés par les efforts internationaux pour mettre fin à l'épidémie de $\mathrm{VIH} /$ sida au cours des 15 dernières années (1) et le Programme commun des Nations Unies sur le VIH/sida (ONUSIDA) a pris l'engagement mondial de mettre fin à l'épidémie de sida d'ici 2030 (2). Depuis que les objectifs de traitement ont été fixés en 2003 , les décès annuels mondiaux liés au sida ont diminué de $43 \%$. Alors qu'environ un million de personnes sont mortes d'un trouble lié au sida en 2016, sur le plan mondial, le nombre de personnes vivant avec le sida et qui en décède est nettement inférieur dans les pays à revenu élevé (3). En partie à cause de la diminution du nombre de cas de sida, certains pays (par exemple, l'Australie) ont cessé d'inclure des données sur le sida dans leurs articles annuels de surveillance $(4,5)$.

L'Agence de la santé publique du Canada (ASPC) travaille en collaboration avec d'autres organismes fédéraux, gouvernements provinciaux et territoriaux, et des organismes 
non gouvernementaux, pour lutter contre le VIH et le sida au Canada. Ce rapport de surveillance est basé sur des articles de cas provenant de nouveaux diagnostics de sida soumis à I'ASPC par toutes les provinces et tous les territoires participants, ainsi que sur des données reçues de Statistique Canada (6). La publication annuelle des données sur le sida fait partie du mandat de I'ASPC de recueillir et d'analyser des données de surveillance à l'échelle nationale, et de publier des rapports à cet effet.

L'objectif de cet article est de fournir un aperçu descriptif des cas de sida signalés par les provinces participantes en cernant les tendances selon l'emplacement géographique, le sexe, le groupe d'âge et la mortalité. Auparavant, les données sur le sida étaient publiées annuellement, avec les données sur le $\mathrm{VIH}$, dans un document autonome intitulé Le VIH et le sida au Canada - Rapport de surveillance. Cet article est la première version du nouveau rapport intitulé Le sida au Canada - Rapport de surveillance 2016 dans le Relevé des maladies transmissibles au Canada (RMTC). Il est à noter qu'un rapport de surveillance distinct sur les cas de VIH, intitulé Le VIH au Canada - Rapport de surveillance 2016, apparaît également dans ce numéro du RMTC (7).

\section{Méthodologie}

Cet article présente des données provenant de deux sources différentes sur le VIH et le sida : le Système national de surveillance du VIH/sida et la base de données Statistique de l'état civil - Base de données sur les décès de Statistique Canada. Le Système national de surveillance du VIH/sida est un système de surveillance passif basé sur des cas, qui rassemble et conserve les données soumises volontairement à l'ASPC par toutes les autorités en matière de santé publique des provinces et territoires. Les détails concernant la collecte des données, la gestion des données, la qualité des données et la classification des groupes de population ont préalablement été décrits (8). En bref, la base de données du Système national de surveillance du $\mathrm{VIH} /$ sida recueille des données non nominatives sur les personnes diagnostiquées du sida et inclut, mais sans s'y limiter, la province ou le territoire déclarant, l'année du test, le groupe d'âge, le sexe, les facteurs de risque (aussi connus sous le nom de catégorie d'exposition) et la race ou l'ethnicité. Dans le présent rapport de surveillance, le terme " cas » ou " cas signalés » désigne les personnes diagnostiquées par une province ou un territoire au cours d'une année donnée et signalées à I'ASPC. Les cas de sida sont comptés selon la date à laquelle l'autorité déclarante a confirmé le diagnostic du sida.

Les cas déclarés de sida doivent correspondre à la définition de cas de surveillance au Canada, tel qu'il est décrit dans la publication du RMTC de l'ASPC intitulée Définitions nosologiques des maladies transmissibles faisant l'objet d'une surveillance nationale (9). Les provinces et les territoires qui fournissent les données au Système national de surveillance du VIH/sida procèdent à l'aide du formulaire de déclaration de cas à l'échelle nationale (8) ou d'une transmission sécurisée de données électroniques. Toutes les données brutes (formulaires papier et ensembles de données électroniques) sont conservées conformément à la directive de l'Agence sur la collecte, I'utilisation et la diffusion de l'information sur la santé publique (ASPC, 2013, document non publié). Les détails concernant les catégories d'exposition du VIH/sida sont disponibles sur le Web exclusif de ce numéro (10).
Les données sur le sida et l'intégralité des données épidémiologiques recueillies et soumises à l'ASPC varient selon la province ou le territoire. En ce qui concerne les rapports sur le sida, certains changements ont eu lieu au fil du temps et affecté l'intégralité des données de surveillance du sida. Les données de l'Ontario sur la catégorie d'exposition et la race ou l'ethnicité n'étaient pas disponibles après 2004 et les données sur le sida au Québec n'étaient pas disponibles après le 30 juin 2003. Le sida n'est plus une maladie à déclaration obligatoire à Terre-Neuve-et-Labrador (depuis 2009) et à l'île-du-Prince-Édouard (depuis 2012). En raison des retards escomptés associés à la déclaration des cas de sida de la Colombie-Britannique, il y a un décalage d'un an dans la publication des données sur le sida (par exemple, les données sur le sida de 2015 ont été publiées dans le rapport de surveillance de 2016). L'information sur la race ou l'ethnicité de la Colombie-Britannique pour les cas de $\mathrm{VIH} /$ sida a été supprimée pour toutes les années, pendant que la province procède à l'examen de cette information.

Les données présentées représentent les cas de sida signalés par les provinces ou territoires participants, diagnostiqués au plus tard le 31 décembre 2016 et qui ont été soumis à l'ASPC au plus tard le 7 juin 2017. Les données ont été extraites de la base de données du Système national de surveillance du VIH/sida plus tard en juin 2017. Des procédures normalisées d'enregistrement de données ont été appliquées à tous les ensembles de données, provinciaux et territoriaux, afin de créer un ensemble de données national aux fins d'analyse. Les analyses descriptives et comparatives portent sur l'année 2016; toutefois, les données de 2015 sont également mises en évidence car, en raison des améliorations apportées à la base de données du Système national de surveillance du $\mathrm{VIH} /$ sida, on n'avait pas demandé aux provinces et territoires de soumettre des données sur le sida pour l'année 2015. Les données des cas signalés depuis le début de la surveillance du sida en 1979 sont également présentées.

Les données complètes concernant les décès annuels dus au $\mathrm{VIH} /$ sida sont disponibles auprès de Statistique Canada (6).

Les logiciels Microsoft Excel 2010 et SAS Enterprise Guide v 5.1 ont été utilisés pour le nettoyage et l'analyse des données. Aucune démarche statistique n'a été utilisée pour les analyses comparatives et aucune technique statistique n'a été appliquée pour tenir compte des données manquantes.

À l'exception des cas où la suppression des données a été demandée par la province ou le territoire, les données dans les tableaux à cellules de petite taille ( $n$ moins de 5 ) n'ont pas été supprimées, puisque la divulgation n'était pas considérée comme un risque qui aurait permis d'identifier des personnes données. Ces procédures sont conformes à la directive de l'Agence sur la collecte, I'utilisation et la diffusion de l'information sur la santé publique de I'ASPC (document non publié). Les données ont été vérifiées par les provinces et les territoires pour assurer leur exactitude. Des informations supplémentaires, y compris des tableaux de données, peuvent être trouvées sur un supplément Web (10)

\section{Résultats}

Le nombre de cas de sida déclarés par année au Canada a diminué de façon constante depuis le milieu des années 1990 (figure 1). En 2016, il y a eu 114 cas de sida signalés à l'ASPC, ce qui est moins élevé que les 190 cas déclarés en 2015. 
Figure 1 : Nombre de cas de sida déclarés par année de diagnostic - Canada, 1979 à 2016

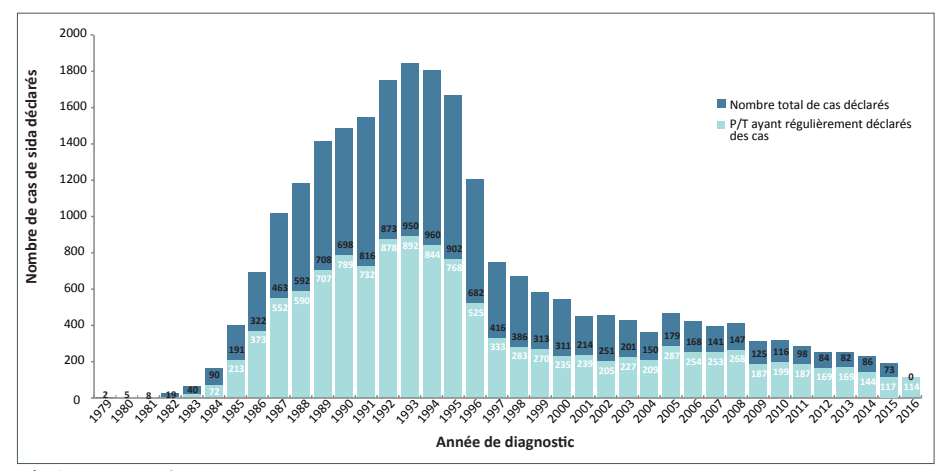

Abréviations : $P / T$, province ou territoire

Légende : La partie supérieure du graphique représente les cas signalés par une $P / T$ qui a cessé de déclarer les cas de sida au fil du temps ou n'a rien déclaré en 2016 : QC (2003), T.-N.-L. (2009) I.-P.-É (2012) et C.-B. (2016). La partie inférieure de chaque barre représente les cas déclarés par les provinces qui ont toujours signalé des cas de sida à l'ASPC depuis 1979

Cependant, il est important de noter que Québec, Terre-Neuve et l'île-du-Prince-Édouard ne déclarent plus les cas de sida et que le nombre de nouveaux cas de sida en 2016 en Colombie-Britannique n'était pas encore disponible au moment de la publication de cet article. Ce nombre de cas de sida déclarés par année représente une diminution de 87,2\% par rapport à l'année 1993, année où l'on a déclaré le nombre le plus élevé de cas $(n=892)$ pour les provinces et territoires qui ont continuellement signalé le sida. De 1979 à la fin de 2016, un total de 24179 cas de sida ont été signalés à l'ASPC.

\section{Répartition géographique}

Parmi les provinces et territoires qui ont déclaré des cas de sida en 2016, les plus grands nombres de cas signalés l'ont été en Ontario $(n=66)$, en Saskatchewan $(n=22)$ et en Alberta $(n=19)$. En 2015, c'est en Colombie-Britannique $(n=73)$, en Ontario $(n=63)$ et en Saskatchewan $(n=29)$ qu'on a déclaré les plus grands nombres de cas de sida (figure 2, tableau supplémentaire 1) (10).

Figure 2 : Nombre de cas de sida diagnostiqués par province et territoire en 2015 et en 2016

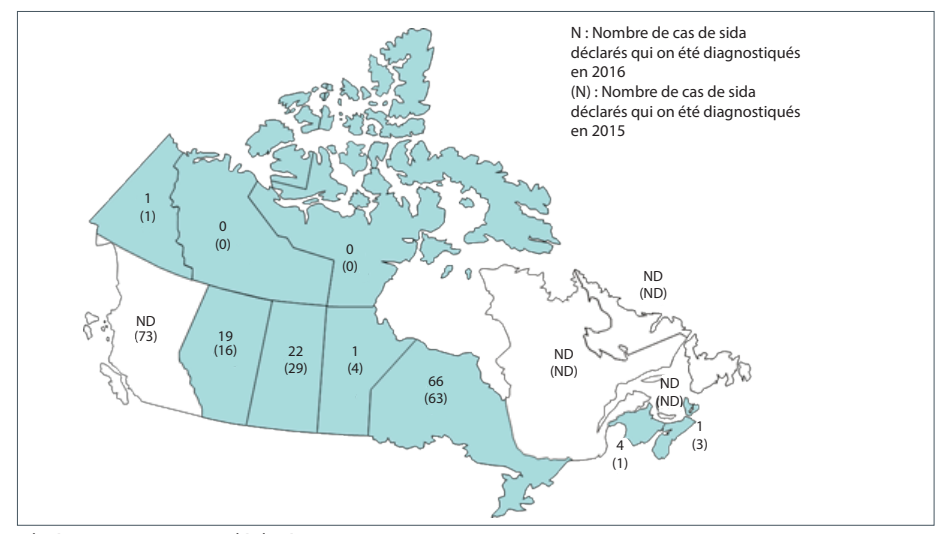

Abréviation : ND, non déclaré

\section{Répartition par âge et par sexe}

Des données sur les groupes d'âge et le sexe étaient disponibles pour tous les cas de sida déclarés en 2016; le plus grand taux se retrouvait parmi le groupe des 50 ans et plus $(36,0 \%)$, suivi des cas signalés dans le groupe des 30 à 39 ans et des 40 à 49 ans $(23,7 \%$ chacun) (figure 3$)$. Un cas de sida a été déclaré chez un enfant de moins d'un an.

Figure 3 : Proportion des diagnostics de sida par année, selon le groupe d'âge -Canada, 2007 à 2016

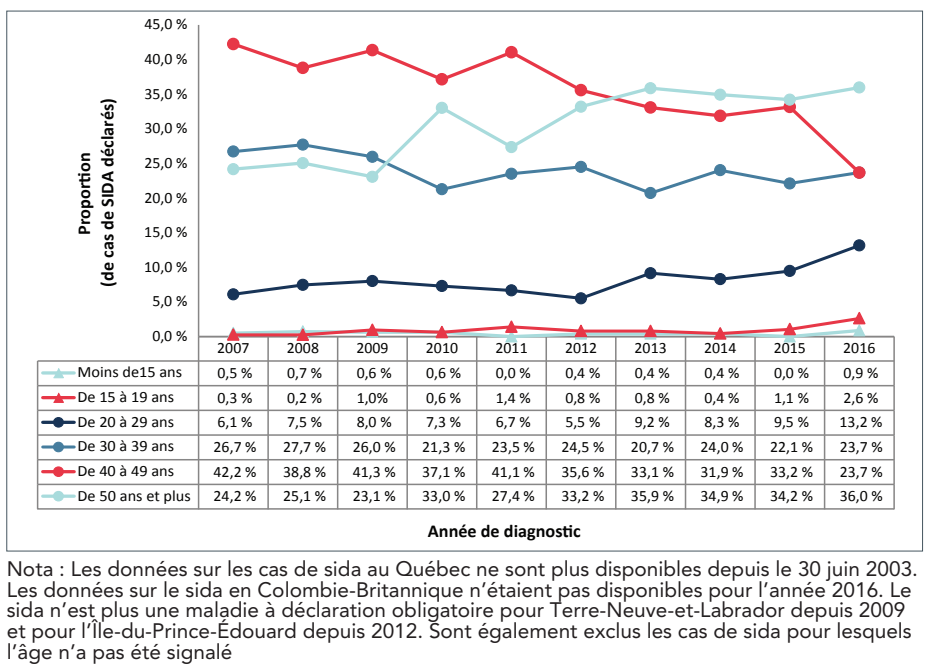

En 2016, la majorité des cas de sida signalés touchaient des hommes (72,8 \%). Parmi toutes les années sur lesquelles portent l'article (1979 à 2016), la répartition par âge des cas de sida est similaire à la répartition par sexe, même si une plus grande proportion de femmes avaient moins de 30 ans (figure 4, tableau supplémentaires 1 à 6) (10).

Figure 4 : Nombre de cas de sida diagnostiqués selon le sexe - Canada, 2007 à 2016

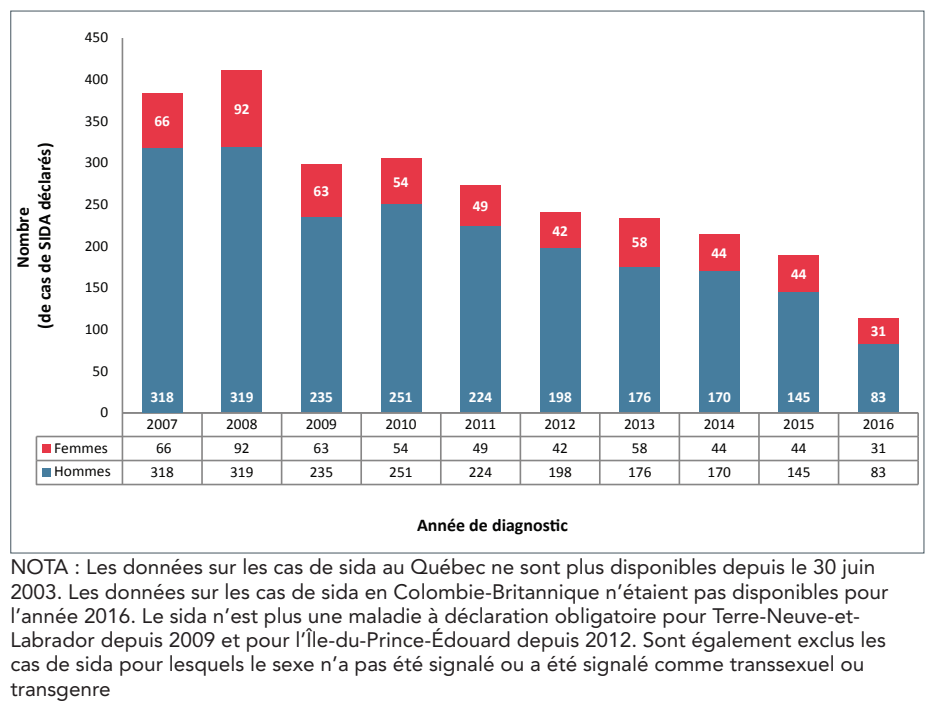

\section{Catégorie d'exposition}

En 2016, 37,7 \% des cas de sida déclarés comportaient des informations sur la catégorie d'exposition. Parmi les données de 2016 disponibles (pour le Yukon, les Territoires du Nord-Ouest, l'Alberta, la Saskatchewan, le Manitoba, la Nouvelle-Écosse et le Nouveau-Brunswick), les proportions les plus élevées de 
catégories d'exposition déclarées chez les adultes étaient les suivantes : utilisation de drogues injectables (UDI) $(35,0 \%)$ et contact sexuel avec une personne à risque (20,0\%). Ces chiffres diffèrent de ceux enregistrés en 2015 (année qui comprend les données de la Colombie-Britannique), où les proportions pour les "hommes ayant des rapports sexuels avec des hommes" et les catégories d'exposition UDI étaient similaires à 23,7\% et $27,8 \%$ respectivement (tableau supplémentaires 7 à 11) (10).

\section{Race et ethnicité}

En 2016, 38,6\% des cas de sida signalés comportaient des informations sur la race ou l'ethnicité. Parmi les données de 2016 disponibles (pour le Yukon, les Territoires du Nord-Ouest, l'Alberta, la Saskatchewan, le Manitoba, la Nouvelle-Écosse et le Nouveau-Brunswick), les plus grandes proportions de races ou ethnicité déclarées, pour tous les groupes d'âge, étaient Autochtones (50,0 \%), Caucasiens (29,5\%) et Afro-Américains $(13,6 \%)$ (tableau supplémentaire 12) (10).

\section{Mortalité due au sida}

Les données fournies sur la base de données Statistique de l'état civil - Base de données sur les décès démontrent que le nombre de décès annuels dus à une infection par VIH a diminué depuis 1995 (figure 5). Le nombre des décès le plus bas attribués à I'infection par VIH ( $n=241)$ a été signalé en 2013, I'année la plus récente pour laquelle des données étaient disponibles. Parmi ces décès liés au sida, il y avait plus d'hommes que de femmes (tableau supplémentaires 13 à 15) (10).

Figure 5 : Nombre de décès dus à l'infection par VIH auprès des adultes (15 ans et plus) selon l'année du décès et le sexe - Canada, 1987 à 2013

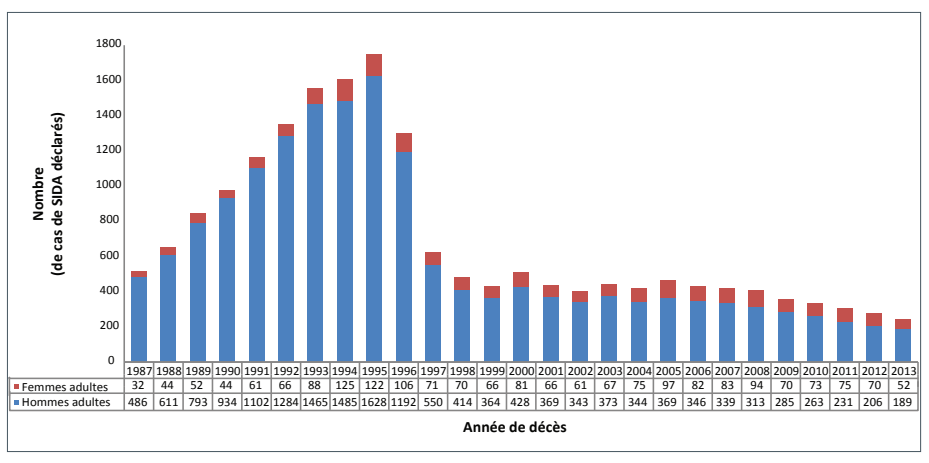

Nota : Données provenant de la Base de données sur les naissances et de la Base de données sur les décès de la Statistique de l'état civil du Canada de Statistique Canada (8)

\section{Discussion}

Le nombre de cas de sida déclarés au Canada a diminué globalement depuis le milieu des années 1990, avec un total de 114 cas de sida déclarés par les provinces et territoires participants en 2016. Il s'agit du nombre le plus faible de cas de sida signalés depuis 1985 (10). Parmi les provinces ayant produit des déclarations en 2016, le nombre le plus élevé de cas de sida a été attribué à l'Ontario $(n=66)$. L'utilisation de drogues injectables $(35,0 \%$ ) et le groupe d'âge des plus de 50 ans $(36,0 \%)$ ont continué d'être la catégorie d'exposition et le groupe d'âge ayant la plus grande proportion de nouveaux cas. En 2016, les cas de sida étaient les plus fréquents chez les hommes (72,8 \%) et les Autochtones (50,0 \%). Il est important de noter que le nombre total de cas de sida pour toutes les années de déclaration tend à surestimer la diminution de l'année la plus élevée (1993) jusqu'à 2016, en raison du nombre de provinces ayant retiré le sida de la liste des maladies à déclaration obligatoire depuis 2003. Dans les provinces qui ont soumis des données sur le sida en 2016, on constate néanmoins une diminution de 87,2 \% des cas de sida entre 1993 et 2016.

À l'exclusion de la Colombie-Britannique $(n=73)$, les comptes les plus élevés de cas de sida en 2015 ont été observés en Ontario $(n=63)$, en Saskatchewan $(n=29)$ et en Alberta $(n=16)$. Aucun changement à cette tendance n'a été noté en 2016, les chiffres les plus élevés ayant été enregistrés en Ontario $(n=66)$, en Saskatchewan $(n=22)$ et en Alberta $(n=19)$. Parmi les cas déclarés en 2016, les adultes de 50 ans et plus représentaient la plus grande proportion de cas de sida $(36,0 \%)$ avec une augmentation au cours des cinq dernières années, proportionnellement aux cas de $\mathrm{VIH}$ nouvellement diagnostiqués dans ce groupe d'âge (11). Au cours de toutes les années sur lesquelles porte l'article, lorsqu'on compare les hommes aux femmes, il y avait une plus grande proportion de cas de sida dans les groupes plus âgés (30 à 50 ans et plus) chez les hommes que chez les femmes, tandis que les femmes représentaient une plus grande proportion des cas dans les groupes plus jeunes (0 à 29 ans). Ces chiffres concordent avec les proportions de groupes d'âge dans les cas de VIH cumulatifs pour toutes les années sur lesquelles porte l'article; cependant, il est possible que la distribution des cas de sida chez les femmes se déplace vers les groupes plus âgés avec le temps, une tendance similaire ayant été notée dans les cas de VIH ces dernières années (11).

Selon l'ensemble de données le plus complet de Statistique Canada, le nombre de décès annuels attribuables à l'infection par VIH au Canada diminue depuis 1995, les données les plus récentes faisant état de 241 décès en 2013, soit le nombre le plus bas à ce jour. Parmi ceux-ci, 78,4 \% étaient des hommes adultes et $21,6 \%$ étaient des femmes adultes, sans décès signalés chez les enfants (moins de 15 ans).

\section{Restrictions et qualité des données}

Les données contenues dans cet article sont considérées comme provisoires et pourraient être assujetties à des changements dans les prochaines versions du rapport de surveillance sur le sida au Canada. Les différences entre les données publiées dans cet article et les données publiées dans les rapports de surveillance nationaux, provinciaux et territoriaux précédents peuvent être attribuables à des retards de déclaration ou à des différences dans la date d'extraction des données des différentes bases de données de surveillance. Lorsque ces différences sont notées, il est recommandé d'utiliser les données des plus récents articles provinciaux et territoriaux.

Plusieurs restrictions doivent être prises en compte. Le Système national de surveillance du $\mathrm{VIH} /$ sida est un système de surveillance passif, basé sur des cas, et il n'est donc pas possible de déterminer si toutes les personnes infectées par le sida sont identifiées et déclarées par les provinces participantes. Les cas de sida peuvent être sous-déclarés pour un certain nombre de raisons. Comme le sida résulte d'une infection par VIH avancée et que le VIH est une infection chronique avec une longue période de latence, de nombreuses personnes nouvellement infectées au cours d'une année peuvent ne pas recevoir de diagnostic de VIH pendant des mois ou des années (12). De plus, 
les provinces et les territoires ne signalent pas tous les cas de sida à I'ASPC et de nombreux médecins omettent de signaler les cas de sida qui touchent les personnes qui vivent déjà avec le $\mathrm{VIH}$. Une deuxième restriction est que trois provinces ne déclarent plus les cas de sida. Troisièmement, les articles ne sont pas toujours complets; par exemple, les données sur la catégorie d'exposition ou l'ethnicité sont soumises pour moins de $40 \%$ des cas. Quatrièmement, les données sur les décès attribués à I'infection par VIH ne sont disponibles qu'à partir de 1987 et les données les plus récentes datent de 2013. Enfin, il est difficile de déterminer et de supprimer les doublons en raison de la nature non nominale des déclarations de $\mathrm{VIH}$ dans certaines provinces et certains territoires. Dans la mesure du possible, les provinces et les territoires examinent et évaluent l'inclusion de rapports en double afin de fournir une image aussi précise que possible. $\mathrm{D}$ 'autres détails concernant les limitations de données ont déjà été publiés (3).

\section{Conclusion}

Le Canada a enregistré des diminutions constantes des cas de sida signalés et des décès liés au sida, l'année 2016 ayant enregistré le compte annuel le plus faible à ce jour pour les deux. Bien qu'il s'agisse là d'une tendance prometteuse, les données doivent être interprétées avec prudence, compte tenu des restrictions de l'ensemble de données, ce qui pourrait entraîner une sous-estimation de l'ampleur de la maladie. En tant que principales sources de données nationales sur les cas de sida, les données contenues dans cet article fournissent de I'information sur les mesures de santé publique et permettent de surveiller les efforts déployés au Canada et à l'échelle mondiale pour mettre fin à l'épidémie de sida d'ici 2030.

\section{Déclaration des auteurs}

L.J. - conceptualisation, logiciel, validation, visualisation, rédaction - ébauche initiale, examen et révision

A.C.B. - rédaction - examen et révision, supervision

M.E. - méthodologie, logiciel, validation, édition des données,

visualisation, rédaction - ébauche initiale, examen

et révision

A.A. - visualisation, rédaction - ébauche initiale, examen et révision

O.V. - visualisation, rédaction - ébauche initiale, examen et révision

W.S. - rédaction - examen et révision, administration du projet, supervision

\section{Conflit d'intérêt}

Aucun.

\section{Remerciements}

L'Agence de la santé publique du Canada aimerait remercier les personnes suivantes des programmes provinciaux et territoriaux de la lutte contre le $\mathrm{VIH} /$ sida pour leur contribution et leur participation :

Sumina Fathima, ministère de la Santé de l'Alberta Rosa Maheden, ministère de la Santé de l'Alberta Mariam Osman, ministère de la Santé de l'Alberta
Services cliniques de prévention, Surveillance et épidémiologie, Centre de contrôle des maladies

de la ColombieBritannique

Carla Loeppky, ministère de la Santé du Manitoba

Rita RaaFat Gad, ministère de la Santé du Nouveau-Brunswick

Shelley Landsburg, ministère de la Santé du Nouveau-Brunswick Sophie Wertz, ministère de la Santé du Nouveau-Brunswick Susan Earles, Santé et services communautaires, Direction de la santé de la population, Terre-Neuve-et-Labrador

Jennifer Phillips, Santé et services communautaires, Direction de la santé de la population, Terre-Neuve-et-Labrador

Heather Hannah, ministère de la Santé et des Services sociaux des Territoires du Nord-Ouest

Angela Fitzgerald, ministère de la Santé et du Mieux-être de la Nouvelle-Écosse

Angie Mullen, ministère de la Santé du Nunavut

Elaine Randell, ministère de la Santé du Nunavut

Abigail Kroch, Réseau ontarien de traitement du VIH

Juan Liu, Santé publique Ontario

Alex Marchand-Austin, Santé publique Ontario

Doug Sider, Santé publique Ontario

Michael Whelan, Santé publique Ontario

Marguerite Cameron, ministère de la Santé et du Mieux-être de l'île-du-Prince-Édouard

Stacey Burns, ministère de la Santé et du Mieux-être de

I'Île-du-Prince-Édouard

Raphaël Bitera, Direction des risques biologiques et de la santé au travail, Institut national de santé publique

du Québec

Micheline Fauvel, Laboratoire de santé publique du Québec, Institut national de santé publique du Québec

Maureen Hastie, Laboratoire de santé publique du Québec, Institut national de santé publique du Québec

Raymond Parent, Direction des risques biologiques et de la santé au travail, Institut national de santé publique du Québec

Diane Sylvain, Laboratoire de santé publique du Québec, Institut national de santé publique du Québec

Helen Bangura, ministère de la Santé de la Saskatchewan John Manalo, ministère de la Santé de la Saskatchewan Lori Strudwick, Centre de lutte contre les maladies transmissibles du Yukon

Rachelle Wallace, Centre de lutte contre les maladies transmissibles du Yukon

Sabrina Plitt, Agence de la santé publique du Canada (Alberta) Elsie Wong, Agence de la santé publique du Canada (Colombie-Britannique)

Suresh Khatkar, Agence de la santé publique du Canada (Manitoba)

Marie LaFreniere, Agence de la santé publique du Canada (Nouvelle-Écosse)

Ashleigh Sullivan, Agence de la santé publique du Canada (Ontario)

\section{References}

1. UNAIDS. Prevention Gap Report. Joint United Nations Programme on HIV/AIDS, Geneva, 2016. http://www. unaids.org/en/resources/documents/2016/prevention-gap

2. UNAIDS. Global AIDS Update 2016. Joint United Nations Programme on HIV/AIDS, Geneva, 2016. http://www. unaids.org/en/resources/documents/2016/Global-AIDSupdate-2016 
3. UNAIDS. AIDS Data. Joint United Nations Programme on HIV/AIDS, Geneva, 2016.

4. Kirby Institute. UNSW. AIDS in Australia is uncommon, but not over. 18 July 2016. https://kirby.unsw.edu.au/news/aidsaustralia-uncommon-not-over. [Consulté sept 2017].

5. Kirby Institute. UNSW. Annual Surveillance Report of HIV, viral hepatitis, STIs 2014. Kirby Institute, Sydney, 2014.

6. Statistiques Canada. Statistique de l'état civil - Base de données sur les décès (BCDECD) (Tableaux 102-0552 and appendice II dans la publication). http://www23.statcan. gc.ca/imdb/p2SV_f.pl?Function=getSurvey\&SDDS=3233

7. Bourgeois AC, Edmunds M, Awan A, Jonah L, Varsaneux O, Siu W. Le VIH au Canada- Tableaux supplémentaires, 2016 (sur le Web seulement). Relevé des maladies transmissibles au Canada. 2017;43(12). https://www.canada.ca/fr/santepublique/services/rapports-publications/releve-maladiestransmissibles-canada-rmtc/numero-mensuel/2017-43/ rmtc-volume-43-12-7-decembre-2017/vih-2016-tableauxsupplementaires.html

8. Agence de la santé publique du Canada. Le VIH et le sida au Canada - Rapport de surveillance en date du 31 décembre 2014, Ottawa, 2015. https://www.canada.ca/fr/santepublique/services/publications/maladies-et-affections/vih-etsida-canada-rapport-surveillance-31-decembre-2014.html

9. Agence de la santé publique du Canada. Définitions nosologiques des maladies transmissibles faisant l'objet d'une surveillance nationale - 2009. Relevé des maladies transmissibles au Canada. 2009;35(S2):1-121. https://www. canada.ca/en/public-health/services/reports-publications/ canada-communicable-disease-report-ccdr/monthlyissue/2009-35/definitions-communicable-diseases-nationalsurveillance.html

10. Jonah L, Bourgeois AC, Edmunds M, Awan A, Varsaneux O, Siu W. Le sida au Canada-Tableaux supplémentaires, 2016 (sur le Web seulement) Relevé des maladies transmissibles au Canada. 2017;43(12). https://www.canada.ca/fr/santepublique/services/rapports-publications/releve-maladiestransmissibles-canada-rmtc/numero-mensuel/2017-43/ rmtc-volume-43-12-7-decembre-2017/sida-2016-tableauxsupplementaires.html

11. Bourgeois AC, Edmunds $M$, Awan A, Jonah L, Varsaneux O, Siu W. Le VIH au Canada - Rapport de surveillance, 2016. Relevé des maladies transmissibles au Canada. 2017;43(12):282-91. https://www.canada.ca/fr/santepublique/services/rapports-publications/releve-maladiestransmissibles-canada-rmtc/numero-mensuel/2017-43/rmtcvolume-43-12-7-decembre-2017/vih-canada-2016.html

12. Hall HI, Halverson J, Wilson DP, Suligoi B, Diez M, Le Vu S et al. Late diagnosis and entry to care after diagnosis of human immunodeficiency virus infection: a country comparison. PLoS One 2013 Nov;8(11):e77763. DOI (http://dx.doi. org/10.1371/journal.pone.0077763). PubMed (https://www. ncbi.nlm.nih.gov/entrez/query.fcgi?cmd=Retrieve\&db=PubM ed\&list_uids=24223724\&dopt=Abstract). 